\title{
DEVELOPMENT AND ANAYSIS OF A CYSTIC FIBROSIS SPECIFIC ANTIBIOGRAM
}

\author{
E. Elson ${ }^{1}$, Ellen Meier ${ }^{1}$, Doug Swanson ${ }^{1}$, Rangaraj Selvarangan ${ }^{1}$, Megan Gripka ${ }^{1}$, and \\ Christopher M. Oermann ${ }^{2}$ \\ ${ }^{1}$ Children's Mercy Hospital \\ ${ }^{2}$ Children's Mercy Kansas City
}

March 23, 2021

\begin{abstract}
BACKGROUND: Antibiotic therapy is essential for the treatment of cystic fibrosis (CF) lung infections. CF-specific airway pathophysiology and frequent antimicrobial exposure increase the risk of resistant infections, creating challenges to antibiotic selection. Antibiotic selection is generally based on previous cultures or hospital-wide antibiograms (HWA); however, most HWA exclude CF isolates. We developed a multi-year CF antibiogram (CFA) to compare with HWA and inform antibiotic selection. METHODS: CF culture data were collected 2015 - 2019 at a single pediatric CF center. All sputum and oropharyngeal swab isolates are included in the CFA. Demographics, microorganism isolates, and susceptibility information are presented. Susceptibilities were reported for methicillin-susceptible Staphylococcus aureus (MSSA), methicillin-resistant Staphylococcus aureus (MRSA), Pseudomonas aeruginosa (PA), Achromobacter species, Burkholderia species and Stenotrophomonas maltophilia. RESULTS: Over five years, the proportion of all SA isolates having methicillin-resistance was higher in the HWA (32\%) than the CFA (28\%). The most common gram-negative CF isolate was PA. Both gram-positive and gram-negative microorganisms were less susceptible in the CFA versus the HWA. CF isolates from sputum were less susceptible than oropharyngeal. MSSA and MRSA had significantly lower clindamycin susceptibility in the CFA compared to the HWA (MSSA $71 \%$ vs $79 \%$, p $<0.0001$ and MRSA $39 \%$ vs $83 \%, \mathrm{p}<0.0001)$. For every antimicrobial tested, PA isolates were less susceptible in the CFA compared to the HWA. There did not appear to be significant changes in susceptibility of CF isolates over time. CONCLUSIONS: These findings have clinical implications for empiric antimicrobial selection. A CFA will allow monitoring of resistance over time.
\end{abstract}

\section{DEVELOPMENT AND ANAYSIS OF A CYSTIC FIBROSIS SPECIFIC ANTIBIOGRAM}

Authors: E. Claire Elson, PharmD, BCPPS ${ }^{1}$; Ellen Meier, CPNP, APRN²; Doug Swanson, MD $^{3}$; Rangaraj Selvarangan, BVSc, $\mathrm{PhD}, \mathrm{D}(\mathrm{ABMM})^{4}$; Megan Gripka, MT(ASCP) $\mathrm{SM}^{4}$; Christopher M Oermann, $\mathrm{MD}^{2}$

${ }^{1}$ Department of Pharmacy, Children's Mercy Kansas City, 2401 Gillham Rd. Kansas City, MO 64108, United States of America

${ }^{2}$ Department of Pulmonary Medicine, Children's Mercy Kansas City, 2401 Gillham Rd. Kansas City, MO 64108, United States of America

${ }^{3}$ Department of Infectious Disease, Children's Mercy Kansas City, 2401 Gillham Rd. Kansas City, MO 64108, United States of America

${ }^{4}$ Department of Pathology and Laboratory Medicine, Children's Mercy Kansas City, 2401 Gillham Rd. Kansas City, MO 64108, United States of America

Corresponding Author: E. Claire Elson, PharmD, BCPPS

Department of Pharmacy 
2401 Gillham Rd. Kansas City, MO 64108

United States of America

ecelson@cmh.edu

Running Title: Cystic Fibrosis Specific Antibiogram

Key Words: Cystic Fibrosis, Pulmonology (general), Antibiotic Therapy

\section{ABSTRACT}

BACKGROUND: Antibiotic therapy is essential for the treatment of cystic fibrosis (CF) lung infections. CF-specific airway pathophysiology and frequent antimicrobial exposure increase the risk of resistant infections, creating challenges to antibiotic selection. Antibiotic selection is generally based on previous cultures or hospital-wide antibiograms (HWA); however, most HWA exclude CF isolates. We developed a multi-year $\mathrm{CF}$ antibiogram (CFA) to compare with HWA and inform antibiotic selection.

METHODS: CF culture data were collected 2015 - 2019 at a single pediatric CF center. All sputum and oropharyngeal swab isolates are included in the CFA. Demographics, microorganism isolates, and susceptibility information are presented. Susceptibilities were reported for methicillin-susceptible Staphylococcus aureus (MSSA), methicillin-resistant Staphylococcus aureus (MRSA),Pseudomonas aeruginosa (PA), Achromobacter species, Burkholderia species and Stenotrophomonas maltophilia.

RESULTS: Over five years, the proportion of all SA isolates having methicillin-resistance was higher in the HWA (32\%) than the CFA (28\%). The most common gram-negative CF isolate was PA. Both gram-positive and gram-negative microorganisms were less susceptible in the CFA versus the HWA. CF isolates from sputum were less susceptible than oropharyngeal. MSSA and MRSA had significantly lower clindamycin susceptibility in the CFA compared to the HWA (MSSA $71 \%$ vs $79 \%, \mathrm{p}<0.0001$ and MRSA $39 \%$ vs $83 \%$, $\mathrm{p}<0.0001)$. For every antimicrobial tested, PA isolates were less susceptible in the CFA compared to the HWA. There did not appear to be significant changes in susceptibility of CF isolates over time.

CONCLUSIONS: These findings have clinical implications for empiric antimicrobial selection. A CFA will allow monitoring of resistance over time.

\section{BACKGROUND}

Individuals with cystic fibrosis $(\mathrm{CF})$ have decreased quantity and/or function of cystic fibrosis transmembrane conductance regulator (CFTR) protein located in cell membranes. ${ }^{1}$ This defect results in abnormal movement of sodium, chloride, and water across epithelial surfaces throughout the body. In the lungs, abnormal airway surface liquid leads to a vicious cycle of inflammation, obstruction, and infection. Infection with methicillinsusceptibleStaphylococcus aureus (MSSA) and methicillin-resistantStaphylococcus aureus (MRSA) tend to predominate early in life but infection shifts with age so that the majority people with CF eventually become infected with chronic Pseudomonas aeruginosa $(\mathrm{PA}){ }^{1,2}$ Antimicrobial therapy is essential in managing acute and chronic CF lung infections. ${ }^{3}$

CF-specific airway pathophysiology and chronic and/or frequent acute antimicrobial exposure lead to clinical challenges when managing infection. Chronic infections and hypoxia increase inflammatory processes which may result in progressive lung damage. ${ }^{4-5} \mathrm{CF}$ lung infection is usually polymicrobial and interactions between microorganisms increase the risk of antimicrobial resistance. ${ }^{4}$ Pseudomonas aeruginosa has increased virulence in $\mathrm{CF}$ lung infection secondary to the development of a mucoid phenotype, alginate biofilms and adaptive resistance mechanisms. ${ }^{5-6}$ These adaptive resistance mechanisms include modifications in targeted binding sites, efflux pumps, and the production of inactivating enzymes. ${ }^{7}$ Additionally, studies have demonstrated there are limitations of oropharyngeal sampling and the challenges associated with this sampling method compared to expectorated sputum. ${ }^{8}$ This may contribute to variability with isolate surveillance and resistance trends over time. The combination of bacterial and host factors decreases the efficacy of treatments by impeding drug delivery, altering pharmacokinetics and increasing the risk of antimicrobial resistance. ${ }^{4,9,10}$ 
Empiric antibiotic selection for the management of pulmonary exacerbations is generally based on hospitalwide antibiograms (HWA). However, most HWA exclude culture antimicrobial susceptibility data from CF isolates. This creates challenges in determining appropriate empiric antibiotic therapy and monitoring for changes in susceptibility patterns over time. This work demonstrates why CF care centers should develop independent $\mathrm{CF}$-specific antibiogram (CFA) for use in clinical practice.

\section{METHODS}

The aim of this study was to develop a CFA at a single pediatric CF care center, Children's Mercy Kansas City (CMKC). IRB approval was obtained at CMKC. Data from all respiratory isolates from individuals with a diagnosis of CF obtained between 01 January 2015 and 31 December 2019 at CMKC were reviewed for study inclusion. Demographic information collected included the sex and age of the patient. Culture source was coded as sputum or oropharyngeal swab and were differentiated through a microbiology report. The microorganisms included in analysis for the CFA included: methicillin-susceptible Staphylococcus aureus (MSSA), methicillin-resistant Staphylococcus aureus (MRSA),Pseudomonas aeruginosa (PA), Achromobacter species,Stenotrophomonas maltophila, and Burkholderia cepaciacomplex including Burkholderia multivorans, B. cenocepacia, B. gladioloi, and B. vietnamiensis species. Per CLSI guidelines, only species with a minimum of 30 isolates during the study period were included in the CFA. ${ }^{11}$ No other isolates were included. Antimicrobial susceptibility information was collected for all CF isolates. Each culture represents a distinct patient encounter. For people with $\mathrm{CF}$ with multiple isolates growing the same microorganism during the same year, only the first chronological isolate was included in the antibiogram. This methodology was based on CLSI guidance for handling multiple isolates within the same year. ${ }^{11}$

The CF antibiogram was compared to the HWA each year. The HWA is an annual compilation of systemwide susceptibility results from cultures from all sources. These HWA are published in June of each year. Cultures from people with CF are excluded from the HWA.

Descriptive statistics included mean and percentages. Additional statistical analyses of antibiogram data and susceptibility information were performed using $\mathrm{X}^{2}$ or Fisher's exact test for categorical data, as appropriate, and t-test for non-categorical data. All tests of significance were two-tailed and $p$-values of [?]0.05 were considered statistically significant.

\section{RESULTS}

Culture results from 373 people with CF were included in the CFA between 2015 and 2019. There were 183 females (49\%) and 190 males (51\%), and the mean age was $12.32+6.43$ years. There were 1,973 culture results representing SA, PA, Achromobacter species,Burkholderia species and Stenotrophomonas maltophiliaincluded in the CFA.

There were 1250 Staphylococcus aureus (SA) isolates; 902 were MSSA (72\%) and 348 MRSA (28\%). SA isolate susceptibility rates between the CFA and HWA were similar except for clindamycin [Table 1]. The CFA demonstrated significantly fewer MRSA (39\% vs. 83\%, p<0.0001) and MSSA ( $71 \%$ vs. $79 \%, \mathrm{p}<0.0001)$ isolates susceptible to clindamycin. Additionally, among the SA isolates collected the HWA demonstrated a higher proportion of MRSA than the CFA (32\% versus $28 \%$ of SA isolates).

When comparing the proportion of methicillin-resistance in SA isolates among sputum versus oropharyngeal swabs in people with CF, there were fewer MSSA isolates obtained from sputum (399, 44\%) compared to oropharyngeal swabs $(503,56 \%)$ and more MRSA isolates, 225 (65\%) and 123 (35\%) respectively. The sputum isolates were less susceptible compared to oropharyngeal isolates for SA [Table 2]. These differences were statistically significant for MSSA isolates from sputum versus oropharyngeal isolates for susceptibility to clindamycin $(\mathrm{p}=0.0202)$.

There were $480 \mathrm{PA}$ isolates from people with CF during the study period [Table 3]. For each antimicrobial tested, CF isolates were less susceptible compared to HWA. Analysis of CF culture susceptibility information based on source, either sputum or oropharyngeal swab, was also completed for PA isolates [Table 4]. There were more PA isolates obtained from sputum $(305,64 \%)$ than from oropharyngeal swabs $(175,36 \%)$ among 
people with CF. Overall, PA isolates from sputum were less susceptible than oropharyngeal isolates. These differences were statistically significant for all antimicrobial's tested except for aztreonam.

Achromobacter species,Burkholderia species and Stenotrophomonas maltophila were also included in the CFA. CFA susceptibility information is summarized in Table 5. Not all isolates have complete susceptibility reported for each antimicrobial presented. Fifty-six Achromobacter species isolates were included in analysis; all but one of these isolates were grown from sputum. ForAchromobacter species, most isolates tested were not susceptible to amikacin $(41,17 \%)$ or cefepime $(39,41 \%)$. Susceptibility to ceftazidime $(56$, $77 \%)$, meropenem $(53,87 \%)$ and trimethoprim/sulfamethoxazole $(55,76 \%)$ were the highest. There were 40 Burkholderia species isolates analyzed; most of the isolates were obtained from sputum ( $\mathrm{n}=32,80 \%)$. The tested isolates were most susceptible to meropenem $(35,83 \%)$ and trimethoprim/sulfamethoxazole $(37$, $78 \%$ ). There were 149 Stenotrophomonas maltophila isolates included most were obtained from sputum $(\mathrm{n}=142,85 \%)$. Twenty percent of the isolates tested were susceptible to ceftazidime $(\mathrm{n}=125)$ and $91 \%$ of the isolates tested were susceptible to trimethoprim/sulfamethoxazole $(\mathrm{n}=148)$. Eleven isolates were tested and susceptible to minocycline $(100 \%)$ and $55 \%(\mathrm{n}=6)$ of the isolates tested were susceptible to levofloxacin.

In addition to analysis of individual isolates, annualized data were characterized to evaluate for potential changes in susceptibility over time. Over the five-year period, there did not appear to be any clinically significant changes in susceptibility patterns for PA, MRSA, and MSSA based on the percentage of overall isolates susceptible to each antimicrobial tested. Susceptibility trends over time are presented in Figure 1. Clindamycin susceptibility trends for MRSA and MSSA are not included in the figure but did not demonstrate significant changes in the CFA over time.

\section{DISCUSSION}

There are no peer reviewed publications regarding CF-specific antibiograms. An abstract published in the proceedings from a conference describes six-years of antimicrobial susceptibility data for MRSA, MSSA and PA from two pediatric CF centers. ${ }^{10}$ Like the current study, they found a higher proportion of MRSA in the HWA. PA susceptibility was significantly decreased compared to the HWA at only one CF site in the abstract. Generalizability is limited based on the relatively small number of isolates analyzed and the susceptibility information presented. The previous abstract and the current study support the development and utilization of CF-specific antibiograms within hospitals and CF care centers.

This study demonstrates that gram-negative and gram-positive microorganisms were less susceptible in people with CF compared to a hospital's general population. The increased resistance seen in the CFA is likely multifactorial, but may relate to increased antimicrobial utilization as well as CF specific differences in airway pathophysiology and microenvironment. ${ }^{1}$ The increase in antimicrobial utilization is driven by systemic therapy for pulmonary exacerbations and chronic maintenance medications with inhaled therapy. ${ }^{2,4,13}$ CFTR dysfunction, airway surface liquid abnormalities and impairment of mucociliary clearance result in increased airway infection. ${ }^{4-7}$ Microorganisms that infect the $\mathrm{CF}$ airway have innate and adaptive resistance mechanisms resulting in decreased susceptibility to antimicrobials. ${ }^{7}$ Alterations in pharmacokinetic parameters of antimicrobials in people with $\mathrm{CF}$ require therapy modification in order to achieve pharmacodynamic targets comparable to healthy populations, resulting in even greater challenges in the treatment of infection. ${ }^{9}$ Demographic information related to the HWA was not obtained, future studies should characterize other populations in order to determine susceptibility differences and trends.

This study is unique in that it highlights the significant differences between sputum and oropharyngeal swab isolates obtained from people with CF. The isolates obtained from sputum were less susceptible than those from oropharyngeal swabs. Multiple factors likely contribute to this finding. Individuals with advanced lung disease are more likely to expectorate sputum and typically have increased lifetime antibiotic exposure, promoting resistance. Presumably, individuals with more advanced age have correlating advanced disease and therefore are able to expectorate sputum resulting in increased resistance. However, this is a major limitation of the present study as age was evaluated as a total CF population rather than culture source. Additionally, previous studies have demonstrated the limitations of oropharyngeal sampling and the chal- 
lenges associated with this sampling method. ${ }^{8}$ For the detection of PA, oropharyngeal sampling compared to bronchoalveolar lavage fluid was more specific than sensitive. For individuals unable to expectorate sputum, the false positive rate was consistently less than $10 \%$ resulting in a high negative predictive value for the presence of PA in oropharyngeal sampling. ${ }^{13}$ However, sensitivity was variable between $44-75 \%$ thus there is a lower positive predictive value for PA in oropharyngeal sampling as well as in the identification of other respiratory isolates. ${ }^{8,12-13}$

Other considerations related to the concordance of sampling includes symptoms and the age of the individual. ${ }^{8,14}$ Despite these limitations, the use of oropharyngeal swabs is standard practice among young, non-expectorating children in CF Care Centers. This may become increasingly relevant in the era of highly effective CFTR modulator therapy, as more people with CF are healthier and unable to produce sputum. ${ }^{15}$ Therefore, understanding the differences in susceptibility between these isolate sources will be even more important when making empiric antimicrobial selections.

This study was reassuring in that there did not appear to be any clinically significant changes in percentage of susceptible isolates during the five-year study period. However, limitations include not standardizing for the number of cultures, source of the culture, or the age of the population. Overall, this study supports the development and sustainment of an institutional CFA.

\section{NOTES}

\section{Acknowledgements.}

The authors would like to acknowledge CMKC pharmacy interns for their contribution to this work: Haley Bettlach, Maggie Bruce, Garret Matthews, Jamie Sullivan, and Brooke Jacobson.

Funding.

No funding has been received for this study.

Potential Conflicts of Interest.

All authors: No reported conflicts of interest. All authors have submitted the ICMJE Form for Disclosure of Potential Conflicts of Interest. Conflicts that the editors consider relevant to the content of the manuscript have been disclosed.

\section{REFERENCES}

1. Goetz D and Ren CL. Review of Cystic Fibrosis. Pediatric Annals. 2019;48(4):e154-e161.

2. Cystic Fibrosis Foundation. Cystic Fibrosis Foundation Patient Registry . 2011 Annual Data Report. Bethesda, Maryland, 2018.

3. Flume PA, Mogayzel PJ Jr, Robinson KA, et al. Clinical Practice Guidelines for Pulmonary Therapies Committee. Cystic fibrosis pulmonary guidelines: treatment of pulmonary exacerbations. Am J Respir Crit Care Med . 2009 Nov 1;180(9):802-8.

4. Döring G, Flume P, Heijerman H, et al. Treatment of lung infection in patients with cystic fibrosis: Current and future strategies. Journal of Cystic Fibrosis. 2012. 11(6): 461-479.

5. Lim S, Fitzgerald D. Treatment resistant Pseudomonas aeruginosa lung disease in young children with cystic fibrosis. Paediatric Respiratory Reviews. 2018 (27): 33-36.

6. Winstanley C, O'Brien A, Brockhurst MA. Pseudomonas aeruginosa Evolutionary Adaptation and Diversification in Cystic Fibrosis Chronic Lung Infections. Trends in Microbiology. 2016. 24 (5): 327337.

7. Stefani S, et al. Relevance of multidrug-resistant Pseudomonas aeruginosa infections in cystic fibrosis. Int J Med Microbiol. 2017: 307(6): 353-362.

8. De Sutter P, Gasthuys E, Van Braeckel E et al. Pharmacokinetics in Patients with Cystic Fibrosis: A Systematic Review of Data Published Between 1999 and 2019. Clin Pharmacokinet (2020).

9. Konold V, et al. Comparison of Population-Specific Cystic Fibrosis Antibiograms Versus Hospital-Wide Antibiograms for Two Cystic Fibrosis Centers. Open Forum Infectious Diseases . 2016;3(1):682. 
10. Hindler JF and Stelling J. Analysis and Presentation of Cumulative Antibiograms: A New Consensus Guideline from the Clinical and Laboratory Standards Institute. Clinical Infectious Diseases 2007; 44:867-73.

11. Eyns H, Piérard D, De Wachter E, et al. Respiratory Bacterial Culture Sampling in Expectorating and Non-expectorating Patients With Cystic Fibrosis. Front Pediatr. 2018;6:403.

12. Seidler D, Griffin M, Nymon A, et al. Throat Swabs and Sputum Culture as Predictors of P. aeruginosa or S. aureus Lung Colonization in Adult Cystic Fibrosis Patients. PLoS ONE. 2016;11(10): e0164232.

13. Mogayzel PJ, Naureckas ET, Robinson KA, et al. Cystic Fibrosis Foundation Pulmonary Guideline: Pharmacologic Approaches to Prevention and Eradication of Pseudomonas aeruginosa Infection. Ann Am Thorac Soc. 2014; 11(10): 1640-1650.

14. Prevaes SM, de Steenhuijsen Piters WA, de Winter-de Groot KM, et al. Concordance between upper and lower airway microbiota in infants with cystic fibrosis. Eur Respir J. 2017 Mar 29;49(3):1602235.

15. Ren CL, Morgan RL, Oermann C, et al. Cystic Fibrosis Pulmonary Guidelines: Use of CFTR Modulator Therapy in Patients with Cystic Fibrosis. Ann Am Thorac Soc . 2018.

\section{Hosted file}

ABGM Tables 03.2021.pdf available at https://authorea.com/users/403588/articles/515001development-and-anaysis-of-a-cystic-fibrosis-specific-antibiogram

Figure 1: Analysis of Susceptibility Patterns Over Time
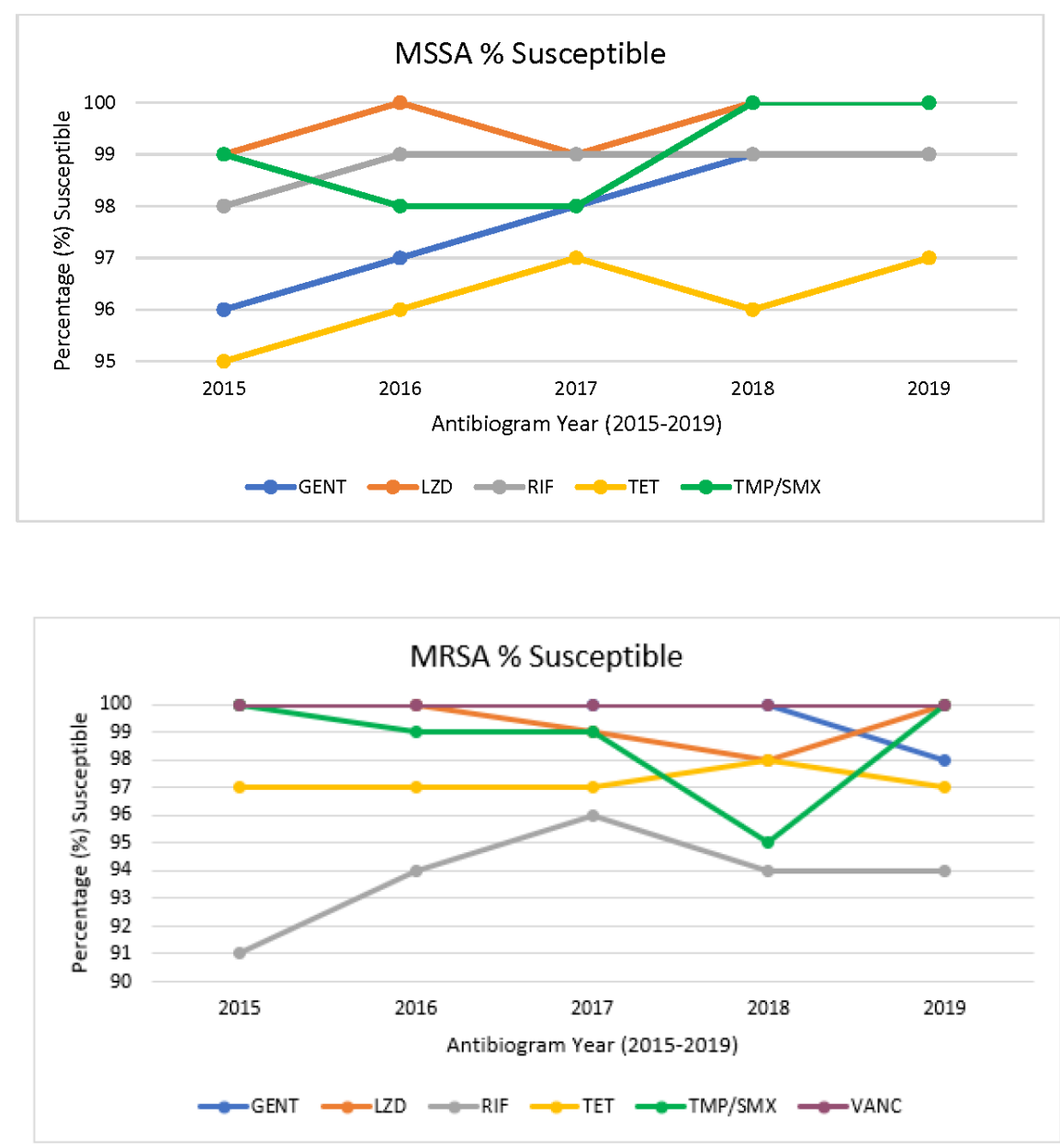


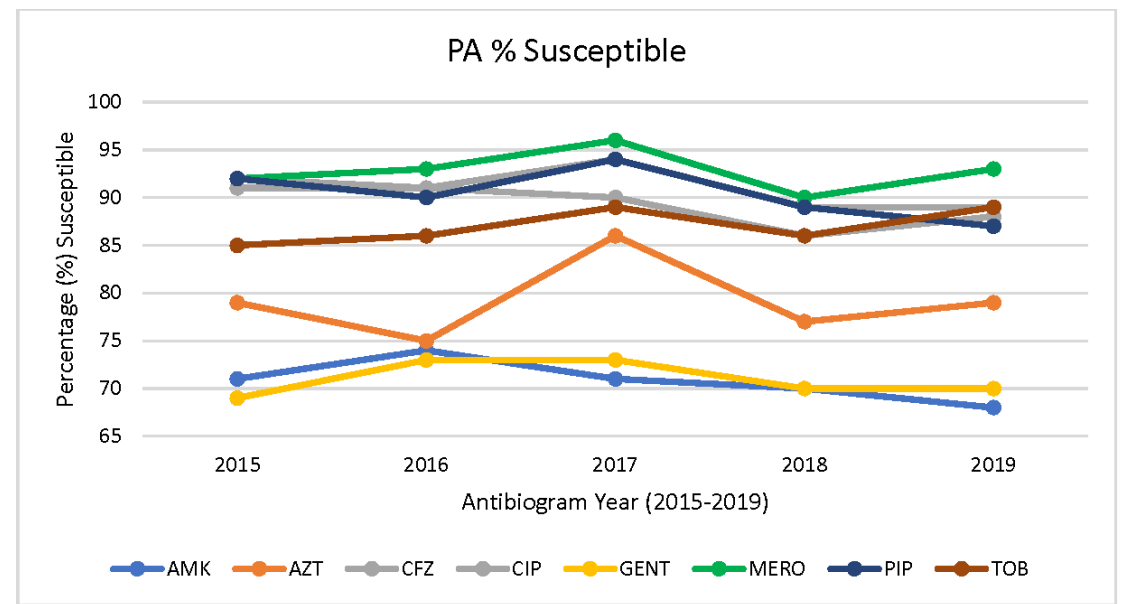

Figure Legend: GENT: gentamicin, LZD: linezolid, RIF: rifampin, TET: tetracycline, TMP/SMX: trimethoprim/sulfamethoxazole, VANC: vancomycin, AMK: amikacin, AZT: aztreonam, CFZ: ceftazidime, CIPRO: ciprofloxacin, MERO: meropenem, PIP: piperacillin/tazobactam, TOB: tobramycin 